\title{
EL DESAFÍO DE LA EDUCACIÓN EN LA NUEVA EVANGELIZACIÓN: LA NECESIDAD DEL YO
}

\author{
Feliciana Merino Escalera \\ Instituto de Filosofia Edith Stein/IAP - Granada
}

\section{RESUMEN / ABSTRACT}

La educación es la atención a la grandeza del misterio humano concreto, de ahí que, el desafío de la educación no consista meramente en la búsqueda de medios eficaces o de sistemas de organización con los que conseguir mejores resultados. Educar es, antes que nada, transmitir algo valioso que conduce al descubrimiento de la vocación propia. Para la consecución de tal fin, el educador no cuenta sólo con la propia fuerza, sino además con la Gracia que procede de Dios.

To teach or bring up is a form of the magnificence of the specific human mystery, therefore, the bringing up challenge is much more than a simply search of efficient ways and management systems for getting better results. To teach is firstly the transmission of something valuable that drives us to the discovery of the own vocation. In order to reach this end, the teacher is not supported only by its own strength but also by the Grace arising from God.

\section{INTRODUCCIÓN}

El tema de la educación nos sitúa de nuevo ante un desafío. No en vano es el tema que la Conferencia Episcopal italiana eligió como principal hasta el 2020 y así nos lo recuerda Benedicto XVI en el discurso que dirigió a sus miembros en mayo de 2010, señalando 
el horizonte que nos proporciona la radicalidad y amplitud de la demanda educativa.

Sin embargo, he de decirles, más bien advertirles, que ustedes, y solo ustedes, han decidido pedirle esta conferencia a una filósofa $\mathrm{y}$ no a una pedagoga. $\mathrm{Y}$ con ello han tenido que asumir ciertos riesgos. Si ustedes hubieran solicitado a un pedagogo que les hablara de educación, les habría hablado de métodos educativos, técnicas eficaces, y teorias pedagógicas, necesarias, a su juicio, y muchas de ellas muy útiles, seguro, para abordar el problema de la educación. Es lógico. Pero yo soy filósofa, o al menos me incluyo entre aquellos que se dedican a la filosofia, y no puedo hablarles de métodos ni técnicas educativas, sino que vengo aquí a ordenar los conceptos, a organizarlos y explicarlos, es decir, a tratar de entender y de explicar en qué consiste y hacia dónde se dirige la educación. Ya saben que si se le pide a un carpintero que haga un coche, es seguro que lo hará de madera.

$\mathrm{Y}$, ¿por qué necesitamos saber en qué consiste y hacia dónde se dirige la educación? Porque no bastan una serie de medios y de herramientas, que sin duda son a la postre necesarias, pues si vamos a emplear una serie de medios, por muy eficaces que sean, tendremos que saber para qué fin. Si desconocemos el fin de lo que hacemos actuamos como el patrón que envía su barco a alta mar dotado de todos los instrumentos de navegación y de avances cientificos de ultimísima tecnologia, pero sin indicarle en qué puerto debe de atracar. Si no conocemos el lugar al que nos dirigimos atracaremos, seguro... en ninguna parte.

A mi juicio, este es el problema fundamental, del que ni siquiera somos conscientes cuando nos dedicamos a hacer reformas educativas. El problema de la educación no consiste en encontrar los medios eficaces o sistemas de organización que aseguren mejores resultados. Y no es que no deseemos la eficacia o la mejor organización, el problema, que a veces ni se plantea en las facultades pedagógicas, el problema que ni entrevemos, es que no sabemos qué significa educar ni para qué educamos.

¿Cómo transmitir lo valioso a los niños si ni siquiera sabemos qué es lo valioso? 
Sabemos hacer caminos, métodos, organismos, instituciones, ningún tiempo ha de ganarnos en nuestra capacidad de organización racional, de concatenación de estructuras y ministerios. Establecemos leyes, reglamentos, órdenes, decretos, y somos capaces de elevar inmensos y complicadísimos procesos en los que están involucradas centenares, miles, millones de personas. Muy bien, pero ¡dense cuenta!, si bien tenemos cohortes de pedagogos, administrativos, psicólogos, políticos, inspectores, secretarios, directores generales, etc., carecemos de una idea clara de lo que estos señores tienen que hacer y, de esta manera, los ponemos a trabajar en una dirección indeterminada a la espera de que se pongan de acuerdo en seguir una línea... que nadie conoce. Porque en nuestro mundo, en nuestra época, en la posmodernidad que se nos ha venido encima, nuestra crisis no es una crisis de estructuras, sino de no saber para qué sirven y a qué han de dedicarse todas esas estructuras que un dia creamos para hacer algo en lo que ahora ya no creemos... sin saber tampoco qué es en lo que tendriamos que creer.

\section{FORMAS DE ENTENDER LA EDUCACIÓN}

Dicho esto, lo que vamos a hacer ahora es organizar el panorama, porque es menester conocer en qué contexto nos encontramos para saber qué es lo que está en crisis, pues solo así podremos comprender cuál es la emergencia a la que nos enfrentamos y recuperar de nuevo el verdadero sentido de la educación.

Ya hace tiempo que venimos diciendo que la educación está en crisis. El maestro está en crisis, el educando está en crisis. ¿Por qué? María Zambrano afirma que hoy día hemos sustituido la vocación por la profesión y la pregunta por el destino por la preocupación por el empleo.

Precisamente estos dos pares de palabras, profesión y empleo, junto a vocación y destino, nos van a ayudar a hacer explícitas las dos formas que tenemos de entender la educación. 


\subsection{Educación proyectiva}

Tenemos una forma de entender la educación que podriamos llamar proyectiva. Proyectar significa, en primer lugar, lanzar, dirigir hacia adelante o a distancia, y en segundo lugar, idear, trazar o proponer el plan y los medios para la ejecución de algo.

Una educación proyectiva busca construir hacia el futuro, y puede estar basada o bien en la sociedad o bien en el sujeto.

\section{A) Proyectiva basada en la sociedad}

Vayamos al primer caso. ¿Qué quiere decir que la educación proyectiva está centrada en la sociedad? A menudo escuchamos que la sociedad del futuro depende de la educación que damos a nuestros hijos hoy, pero tal vez no nos damos cuenta del alcance de esta afirmación. Existen personas, que hacen leyes e idean programas educativos, que tienen una idea más o menos clara de la sociedad que desean construir en el futuro, y establecen un modelo educativo cuya función es modelar a los niños para que sean ellos los instrumentos de transformación del mundo, de construcción de la nueva sociedad pensada por adelantado.

Por ejemplo, si sabemos que la sociedad del futuro va a ser una sociedad en la que los sujetos van a pasar entre 10 y quince horas sentados en un ordenador, entonces necesitaremos fisioterapeutas, así que creamos la licenciatura para que estudien fisioterapia. O si el inglés va a ser la lengua fundamental en el futuro, pues hagamos que estudien inglés desde pequeños, convirtamos los centros en centros bilingües, y a los profesores exijámosles que se saquen el first o los títulos que hagan falta para poder enseñar inglés.

Este modelo proyectivo basado en la sociedad lo que consigue es, en primer lugar, moldear (o manipular podríamos decir también en el sentido latino de "manipulus", el que trae a su mano) a los jóvenes aplicando un criterio, el criterio de cómo se desea que sea la sociedad del futuro, para a través de él modelar a las nuevas generaciones. Es decir, modela a los jóvenes para cambiar el futuro desde el punto de vista de los valores; en segundo lugar intenta, proyectando cómo será la sociedad del futuro, enseñarles formación 
profesional, es decir, aquello que va a ser útil en el futuro. Desde el punto de vista de la capacitación, piensa qué va a ser importante y necesario en el futuro y se lo enseña ahora a los jóvenes.

Por ejemplo, se les educa en los valores del ecologismo: el reciclaje, la importancia de las energías renovables, etc, o en la importancia de la paz, la ideología de género, etc, porque es el criterio para proyectar la sociedad que se desea en el futuro, y en cuanto a la cualificación concreta, se les pide que estudien inglés, o fisioterapia o experto en gestión de energias renovables.

Dense cuenta de que este modelo de comprensión de la educación no es necesariamente malo. De hecho, tiene la virtud de conocer aquello que se desea hacer, el fin que se quiere alcanzar. La validez o no del proyecto educativo que se impone a los jóvenes tendrá que ver con la concepción antropológica y teológica que la fundamente (porque toda concepción del hombre, por muy atea que se afirme, es una forma implícita o explícita de teología). La validez de la educación de hoy tendrá como primer criterio la validez del modelo humano y social que se propone.

\section{B) Proyectiva basada en el sujeto, en la persona}

En el segundo caso, ¿en qué consiste una educación proyectiva basada en el sujeto individual, en la persona?

Es evidente que la persona individual, desde que nace, muestra unas habilidades y talentos, lo que llamamos dones naturales, es decir, aquello para lo que realmente está capacitado. La educación proyectiva consiste en el desarrollo de las capacidades propias del sujeto individual, en la observación de sus potencialidades y talentos concretos, al tiempo que de sus deseos y preferencias. Se trata de observar cuáles son esas capacidades, preferencias y deseos, que normalmente suelen acabar por coincidir. Por ejemplo si uno ve que un joven alumno está dotado para la pintura, suele pasar también que le gusta pintar, y su don le sirve como estímulo y motivación para pintar, puesto que ve que lo hace bien. En definitiva, se trata de atender a su vocación, dejar que el sujeto se exprese libremente desde aquello que por naturaleza le ha sido dado como don natural, para así lograr que dé lo mejor de sí 
mismo en función de sus potencias e intereses, desde un proyecto de sujeto individual, que ve en él la semilla que bien cuidada llegará a ser árbol, es decir, el proyecto de hombre que bien encaminado, le convertirá en la mejor versión de sí mismo. Esto puede ocurrir, tanto porque se utilice un método por el que sólo se puede aprender aquello que se hace, es decir, la pura práctica, tal como defiende el pragmatismo, o también puede ocurrir a través del acompañamiento de un maestro que lo orienta en su vocación y dirige su formación. No es esto lo que ahora nos preocupa, sino el hecho de que esta manera de educar tiene en el sujeto individual la respuesta al éxito de la educación.

\subsection{Educación reflexiva o adaptativa}

Hasta aquí todo lo referente a la primera forma de entender la educación basada en el proyecto de sociedad o de hombre.

Pero hemos dicho que hay una segunda forma de entender la educación que es la reflexiva o adaptativa.

En esta, partimos del mundo tal y como es, el mundo entendido como una red de estructuras, de instituciones, un sistema económico, un sistema político, una concepción de la verdad, del bien, con su propio sistema de organización administrativa, jurídica y policial, etc, y todo aquello que sabemos que forma parte de lo que es el mundo tal y como nos es dado, también en lo referente al sistema social predominante. De lo que se trata es de convertir el sistema educativo en una especie de entrenamiento para la adaptación de los sujetos a dicho sistema.

Me explico, esta forma de entender la educación lo que hace es considerar el modelo educativo a imagen y semejanza del modelo social imperante y establece los procesos y métodos en el ámbito educativo a imagen de los que se considera que socialmente conducen al éxito: así los procedimientos organizativos, los tiempos requeridos, los niveles exigidos, los requisitos de acceso al mundo laboral, todo ello según los criterios de la sociedad reinante, de modo que educamos al sujeto de la mejor manera para que finalmente pueda adaptarse y tener éxito en dicho entorno. 
Este modelo reflexivo es conductista, pues una vez se conoce y comprende cuál es el modelo, la cuestión es cómo provocar en el educando la adaptación óptima a dicho modelo, de modo que la educación tiene como único objetivo repetir el modelo.

Todas las sociedades que no tienen claro lo que significa educar siguen o tienden a seguir este sistema adaptativo o reflexivo, porque si no existe un criterio, la alternativa es precisamente la repetición de la mentalidad dominante, lo que predomina, la reproducción del modelo.

También es lógico que sea así, ya que tal y como vimos en el modelo proyectivo basado en la sociedad ¿quién tiene una bola de cristal para adivinar lo que va a ser y cómo va a ser la sociedad en el futuro? Nadie tiene una bola o varita mágica. Por ello tendemos a ser conservadores y educamos según los cánones por los que se rige la sociedad en el presente, para no arriesgar. Por ejemplo, le decimos a nuestro hijo: estudia derecho porque es una licenciatura universitaria que tiene actualmente muchísimas salidas profesionales.

Por otro lado, tenemos también miedo de que fomentar las particularidades individuales, los talentos, la vocación concreta, tal como apuntamos que sucede en la educación proyectiva basada en el sujeto individual, tenga como consecuencia crear inadaptados sociales, entre otras cosas porque ¿cómo conseguimos fomentar las vocaciones y talentos individuales? Ni más ni menos que según los estándares de excelencia establecidos.

Así, por ejemplo, si a un chaval lo que le gusta es construir casas, estudiará arquitectura, y si lo que hace bien es pintar o tocar un instrumento, tendrá que acometer el aprendizaje durante años en el conservatorio o escuela de artes, porque los niveles son muy complicados y requieren de un aprendizaje muy extenso en el tiempo que además no aseguran el éxito. Al final la educación que se centra en la vocación del sujeto individual también tiene que adaptarse a los estándares profesionales imperantes.

No obstante, si no fomentamos las vocaciones en nuestros hijos, entonces lo que hacemos es animarlos a ser mediocres, a 
ser abogados sin vocación, economistas sin vocación o agentes de bolsa sin vocación.

Tendemos a ser conservadores, adaptativos, en la medida en que llevamos a la educación siempre por detrás de los sistemas sociales vigentes. Por eso constantemente escuchamos voces que dicen que el futuro de nuestra sociedad dependerá de cómo eduquemos a nuestros hijos, como ya vimos, pero en realidad los educamos reiterando las formas y estructuras del discurso dominante, que además se ha vuelto acrítico, porque no se cuestiona. Es la educación una versión o corolario del darwinismo social: la adaptación al modelo establecido y el éxito que sólo dicha adaptación puede producir. El sujeto, asi, es educado para convertirse en el engranaje de un modelo social fijado previamente.

\section{NUESTRO MODELO EDUCATIVO}

¿Cuál es el modelo educativo que tenemos aquí en España?

Nuestro modelo educativo actual responde esencialmente a los parámetros del modelo reflexivo o adaptativo.

Nuestro sistema económico tiene como base una concepción competitiva del tiempo y del esfuerzo: hay que conseguir hacer cada vez más en menos tiempo y a ese parámetro se le denomina productividad. Este concepto fue un invento de un obrero espabilado llamado Frederick Taylor, que ideó un sistema para racionalizar la producción (con un concepto de razón basado en la eficiencia a cualquier precio). La idea de Taylor consistía en dividir los trabajos necesarios para realizar un equipamiento en acciones simples, y en dedicar un operario a esa acción. A esto se le denominó "cadena de montaje". Después cronometró el tiempo que un operario tardaba en realizar una operación, y adjudicó el salario base al hombre que lograse el tiempo récord. Quien tardase más, y de esta manera retrasase a la cadena, ganaría menos, quien superara el record ganaría más... ese dia, porque habría establecido una nueva marca que determinaría el salario base.

Nosotros hemos estado utilizando un modelo similar para programar el sistema educativo. Se divide a los chicos en edades, 
por lotes, da igual la capacidad que tengan, su formación previa o sus habilidades. Es necesario basarse en un criterio "objetivo". Se divide lo que tienen que aprender en pasos sencillos, en asignaturas $\mathrm{y}$ en módulos temporales u horas, y se intenta que aprendan lo máximo en el menor tiempo posible. Tratamos a los alumnos como si fuesen unidades al servicio del sistema económico. Este modelo, que predominó en Europa hasta los años ochenta, fue después moderado por otro principio contrario. Evidentemente, al igual que el taylorismo, la educación imponía un modelo competitivo. Existían profesores que valoraban a los alumnos según un sistema denominado "la campana de Gauss", que sitúa el cinco en la nota media de la clase, con lo que una persona que sacara un ocho solo alcanzaba un cinco si la media de la clase era de ocho. Un modelo extraordinariamente competitivo. Como es lógico el modelo desestima a quienes tienen habilidades distintas de las que prima el sistema o a quienes aprenden más despacio de la media.

Es este un modelo, por igualitario, discriminatorio en el que se enseñan las aptitudes meramente instrumentales donde lo que importa es que una persona sepa utilizar su razón lógica, discursiva (lo que precisa de tiempo) para obtener unos resultados determinados en un tiempo determinado, porque eso producirá éxito académico. Es evidente, que en el caso de las competencias artísticas y humanas, cada vez más en declive, la eficiencia y el pensamiento lógico-deductivo no son suficientes, ni se aprenden en el mismo tiempo (el tiempo del desarrollo individual, que no siempre es lineal ni ascendente) Así, si alguien en el grupo va más lento, hace que la cadena de producción, como ocurre en el sistema taylorista, vaya más lenta, por lo que suele ocurrir que el que sigue el ritmo permanece en la clase, y el que no queda fuera. ¿Qué consecuencias tiene esto? Tratar a los alumnos como meros instrumentos para el logro de los máximos resultados en el menor tiempo posible. Y ese fin lleva, o a eliminar a los sujetos que no están al mismo nivel y siguen el mismo ritmo, o a adaptar tu ritmo a los más lentos, lo que también lleva a resultados perniciosos por una igualdad malentendida, provocada precisamente por esta división en módulos rígidos.

Un claro ejemplo de este modelo que estamos explicando lo podemos ver en la revista Forbes. La revista Forbes realiza cada año un listado de las mejores escuelas de negocio según un criterio 
denominado "tasa de retorno de inversión", consistente en calcular cuál es el salario que cabe esperar si uno estudia determinada cosa en según qué sitio, menos el coste de los estudios y el lucro cesante, es decir, el dinero que dejo de ingresar si me dedico a tiempo completo a estudiar y ceso en mi ocupación previa. He aquí un sistema educativo claro, en el que se sabe muy bien cuál es el objetivo de la educación: ganar dinero. Fíjense que una vez que hemos determinado cuál es el objetivo del sistema educativo ya no resulta tan complicado, de hecho es en esto en lo que somos expertos, regular, organizar, estructurar y determinar cómo y en qué van a usar su tiempo los estudiantes. Quizás el problema sea establecer sin ningún tipo de duda cuál es ese fin tan importante y decisivo para el que se van a preparar durante tanto tiempo y de forma tan incisiva nuestros estudiantes, nuestros hijos, nuestros jóvenes. Se podrá decir que entonces basta con establecer un fin y que bueno será el que la revista Forbes establece, que no es otro sino el quién es quién cuando el qué es el dinero. Sin embargo, el salario es una cosa que solo cobra sentido en un universo de relaciones y comparaciones, con lo que actualmente no somos ricos por ganar mucho, sino por ganar más que los demás, más que los otros.

E1 socialismo ha venido a atemperar estos excesos, trayéndonos otros. En aras de la igualdad se han decidido eliminar los términos competitivos. En lugar de andar todos en pos del primero, vamos a andar todos en pos del último, ¡él será ahora quien marque el ritmo! Además tiende a ser más incisivo en la formación de valores, pero como es dominante lo que hace es utilizar la educación para reproducir los valores de la mentalidad dominante (lo hemos dicho ya, ideología de género, ecologismo, no violencia, tolerancia, etc). Queriendo ser proyectivo, como al final es un discurso dominante, se ha vuelto conservador, convirtiéndose en un aderezo del capitalismo. Es capitalismo en lo económico y social, pero aderezado del ideario socialista. En este sentido, es lógico que tenga que prolongarse el proceso formativo de los muchachos, porque en las primeras etapas se enseñan básica y fundamentalmente educación para la ciudadanía bajo la apariencia de diferentes asignaturas (lo que también sucede en las escuelas concertadas), teniendo que ser los padres los que enseñen los contenidos formativos en casa.Por ello, lo volvemos a repetir, es un modelo desigualitario, discriminatorio. 
Los que progresan lo hacen o porque sus padres disponen de mucho tiempo libre o porque no teniéndolo se pueden permitir pagar un profesor particular que enseñe a sus hijos los contenidos concretos. Esto es un hecho. Los padres tenemos que enseñar a nuestros hijos en casa porque vienen del colegio sin saber lo mínimo necesario. Ahora, eso sí, saben muy bien lo que es el Día de la Paz, qué es el reciclaje, etc. Muchos de estos principios serán muy buenos, pero las matemáticas se las enseñamos su padre y yo en casa. Y esto nos tiene a los padres bajo un permanente asombro.

\section{UNA PROPUESTA DE EDUCACIÓN EN EL CONTEXTO DE LA NUEVA EVANGELIZACIÓN}

Esta es la situación que ha llevado a Benedicto XVI a hablar de una verdadera emergencia educativa: desconocemos qué es la educación y para qué se educa, por lo que convertimos nuestras escuelas en academias de formación capitalista, academias que por otra parte, vivimos y fomentamos también en los hogares cuando educamos a nuestros hijos.

Resumiendo, esta emergencia puede centrarse en dos aspectos:

1) La adaptación a un modelo social basado en la obtención del dinero como símbolo de éxito social, con lo que lo que era un simple medio se ha convertido en el fin de la educación.

2) El relativismo como propuesta ideológica que nunca es verdadero, porque en realidad es la máscara que utiliza el poder para imponer sus pretensiones ideológicas.

Esta manera de entender la educación sólo puede dejar insatisfecho, porque como dijo Benedicto XVI en el discurso que pronunció en 2007 en la basílica de San Juan de Letrán, por un lado se transmiten determinadas habilidades o capacidades de hacer, mientras por otro se busca satisfacer el anhelo de vida y el deseo de felicidad de los jóvenes a través de los objetos de consumo.

¿Cuál es entonces la respuesta adecuada a esta emergencia educativa, que tiende a reproducir una sociedad injusta al tiempo 
que la vacía de contenido? La respuesta es despertar el yo, porque la finalidad esencial de la educación, siguiendo a Benedicto XVI, es "la formación de la persona a fin de capacitarla para vivir con plenitud y aportar su contribución al bien de la comunidad."1

En palabras de la santa y patrona de Europa, Edith Stein, Santa Teresa Benedicta de la Cruz, que fue filósofa y educadora entre otras muchas cosas, esto puede traducirse con una palabra: vocación, o con dos: vocación y profesión, con lo que volveríamos a unir los pares de palabras que al principio, citando a María Zambrano, quedaban divididos.

De hecho, El término alemán Beruf se traduce doblemente por "profesión" y por "vocación". Cuando Edith Stein analiza el significado del término Beruf observa que "en el uso coloquial habitual tiene la palabra Beruf un sentido muy debilitado, que apenas si permite aún traslucir algo de su originario significado. Cuando los niños han llegado al último curso de la escuela se comienza a pensar qué profesión (Beruf) han de escoger; se ha discutido mucho si las mujeres han de entrar en la vida profesional (Berufsleben) o si deben quedarse en casa. Al respecto, bajo el término Beruf se entiende la mayoría de las veces no mucho más que una actividad laboral. Sólo en algunas circunstancias se mantiene todavía el sentido originario de la palabra en cuestión; así, cuando de alguien se dice que ha perdido su vocación (Beruf), o cuando se habla de la vocación religiosa (Klosterberuf). En ambos casos se está manifestando que Beruf es algo para lo que uno debería estar llamado (berufen sein)". ${ }^{2}$

Hoy es cada vez mayor la atención que se dedica en el ámbito pedagógico a la vocación. Se insiste en que los chicos estudien algo para lo que se sientan llamados, se realizan tests de orientación

1 Benedicto XVI, Discurso a la asamblea diocesana de Roma sobre el tema "Jesús es el Señor. Educar en la fe, en el seguimiento y en el testimonio", 11 de junio de 2007 en la Basílica San Juan de Letrán.

2 Edith Stein, "Vocación del hombre y de la mujer según el orden de la naturaleza y de la gracia", en Obras completas, IV: escritos antropológicos y pedagógicos, Monte Carmelo/El Carmen/Espiritualidad, Burgos/Vitoria/Madrid, 2003, p.272 (En adelante, citamos las obras completas como OC, añadiendo el tomo correspondiente). 
vocacional y es el deseo de todo estudiante elegir una profesión en virtud de su vocación. La vocación, sin embargo, acaba ocultando un enfoque instrumental en un contexto mercantilista, limitándose a ser una cualidad adjetiva -y deseable- en toda profesión, pero no un principio configurador de la propia vida. ${ }^{3}$ Así lo expresa Stein: "Quien considere su trabajo como simple fuente de ganancia o como pasatiempo lo desarrollará de una forma completamente distinta de aquel para quien sea "vocación profesional" en sentido propio, es decir, de aquel que se sienta llamado a ello". "Y ese es el carácter, el ethos propio de la vocación: "la actitud animica duradera o totalidad de hábitos que en la vida profesional de un ser humano se presentan desde su interior como principio configurador". ${ }^{5}$ No se trata de educar sólo para hacer buenos profesionales que respondan a las exigencias del mercado, sino de educar para algo más profundo como es hacerse cargo de la realidad y de la propia vida, como exigencia de significado de la propia existencia. ${ }^{6}$ "Estamos en el mundo para servir a la humanidad", afirma la propia Stein. No estamos al servicio del mercado o del Estado, por ello ser serios con la vocación supone comprender mejor el profundo significado que tiene el trabajo para nuestra vida, dificilmente reducible a términos instrumentales.

Hoy día nos acostumbramos cada vez más a un modelo de relaciones en las que prima la lógica del mercado y la mentalidad empresarial. Es la misma lógica que nos obliga a estudiar hasta la treintena o más bajo un proyecto educativo que nos define como "útiles" para poder ocupar un puesto de trabajo que no siempre resulta digno dentro del sistema capitalista, la que marca la orientación de nuestra profesión decidiendo por nosotros qué itinerario profesional debemos seguir en función de las necesidades del mercado, la que nos obliga después a retrasar nuestro proyecto vital, rechazándonos sin disimulos cuando queremos cumplir con

3 "Y así, en vez de vocación se habla de profesión, despojando a esta palabra de su primordial sentido, haciéndola de equivalente de ocupación o de simple trabajar para ganarse la vida", Maria Zambrano, Filosofia y educación, op. cit. P. 101

4 Edith Stein, "El ethos de las profesiones femeninas", en OC, IV, p. 162.

5 Ibid.

6 Sobre ello, vid. Javier Barraca, Vocación y persona. Ensayo de una filosofía de la vocación, Unión editorial, Madrid, 2003, pp. 197 y ss. 
nuestra "vocación" -y digo bien-de madres, la misma que permite el aborto por causas económicas y sociales en pro de la productividad, la misma que nos facilita todo tipo de servicios sociales para que conciliar la vida laboral y familiar signifique dejar a nuestros hijos en guarderías estatales diez horas al día para que podamos trabajar más y mejor. Hemos conseguido manejar a las personas como si se tratara de bienes útiles, instrumentalizando otro tipo de bienes que constituyen una fuente de riqueza para la vida comunitaria, pero que no pueden desarrollarse en una lógica instrumental porque sólo son concebibles desde una óptica sacramental. Hablo, por ejemplo, de la familia.

Una visión del trabajo que perdiera su carácter humano lo vaciaría de significado, cuyos fines no se limitan exclusivamente a la ganancia o retribución económica, sino que son también fines del trabajo aquellos que redundan en mejorar el mundo, la vida y a nosotros mismos ${ }^{7}$. Con el término Beruf, se hace explícita la conexión vital entre vocación y profesión, creatividad y actividad.

El nexo entre trabajo y riqueza, tan difundido en esta actualidad de crisis económica y financiera, refleja que el trabajo es un bien escaso. Sin embargo, no es tan cierto que el trabajo sea un bien escaso, sino que está mal compartido. Muchos ya han hecho este diagnóstico. "En primer lugar, en el sentido de ese trabajo: trabajamos mal porque hemos perdido la razón de por qué y para qué trabajamos. En segundo lugar en la delimitación de la acción humana que es considerada como trabajo y la que no: sólo es trabajo el manejo de cosas; no lo es en muchas ocasiones el cuidado de las personas. En tercer lugar, en la atribución de beneficios económicos a ese trabajo: los canales de economía no traducen en empleo todo el trabajo beneficioso para la comunidad, mientras las grandes cifras de capital flotante juegan arbitrariamente con los destinos de las personas y las colectividades". ${ }^{8}$

YEPES, R., Fundamentos de antropología. Un ideal de la excelencia humana, EUNSA, Pamplona 1996, p. 334.

8 PERIS, J. A., "El trabajo y la vida familiar", conferencia inédita, Encuentro de familias por el Reino de Cristo, Madrid, 1998, p. 5. 
La personalización ${ }^{9}$ del trabajo constituye un tema fundamental por la llamada (vocatio) a toda la sociedad a basar las relaciones laborales no en el dinero o el éxito, sino en otros principios, como el cuidado, la asistencia y protección, el servicio y colaboración mutua. Al fin y al cabo, el trabajo no es sino una expresión de la vocación de entrega del ser humano.

La vocación tiene por tanto sentido en diversos órdenes: el de la corporalidad, el de los estados de vida (el matrimonio, la vida consagrada, etc), el del trabajo. Se trata de la llamada al cumplimiento de la vida, que es Cristo, que se expresa en diversos órdenes.

Por ello, la palabra que mejor responde a la superación de la crisis educativa, es la de vocación. Vocación significa llamada. Procede de vocare, y hace referencia tanto a la acción de llamar como al hecho de ser llamado, expresando la profunda unidad entre la llamada y la realización de aquello que responde a esa llamada. Sin embargo, se trata de una llamada singular, que conecta al ser humano con el origen mismo de la llamada. ${ }^{10}$ Lo expresa muy bien Jean Lacroix, cuando dice que "si cada persona tiene una vocación, su realidad no puede residir más que en la respuesta que da a esa llamada que Dios le hace oír continuamente llamándole por su nombre. Tal es, como se sabe, el punto de vista de Kierkegaard al mostrar que mi vocación, que es mi mismo ser, es una palabra personal que Dios me dirige llamándome por mi nombre". ${ }^{11} \mathrm{O}$ también Emmanuel Mounier, quien afirma: "La llamada permanente de la vocación implica una actitud permanente de ruptura respecto a todo lo

9 En este sentido mounier, E.: "Todo trabajo trabaja por hacer un hombre al mismo tiempo que por hacer una cosa", Obras Completas, II, Sígueme, Salamanca 1993, p. 355. Fundamental es también la obra de wOJTYLA, K., Persona y acción, Biblioteca de Autores cristianos, Madrid 1982. En ella, siguiendo la teoría fenomenológica de los valores de Scheler, analiza el significado personalista de la acción humana como autorrealización, como valor esencial: "El valor personalista, por otra parte, es intrinseco a la misma realización de la acción por la persona, al hecho mismo de que el hombre actúe de forma adecuada a lo que es él mismo, de que la autodeterminación sea inherente auténticamente a la naturaleza de su actuar -realizándose la transcendencia de la persona por medio de su actuación”, op. cit., p. 309.

10 Sobre el concepto de vocación y su importancia para la filosofia, vid. el ensayo de Javier Barraca, Vocación y persona..., op. cit.

11 Jean Lacroix, Amor y persona, Caparrós, Madrid 1996, p. 38. 
que pueda ahogar su voz o desviar su sentido: ruidos del mundo, egoísmos de las familias, conformismos públicos, usurpaciones de las colectividades que se arrogarian sobre esta intocable un derecho de inspección y de dirección. Lo cual no es aún más que una condición negativa para la libertad de la vocación; ella pide algo más que protecciones: pide esa atención del corazón, ese hábito de recogimiento, que educa la virtud natural y sobrenatural del silencio a fin de que, de milagro en milagro, me acerque a este intimius intimo meo, a este corazón inaccesible de mi corazón, donde Dios 'desde el seno de mi madre... me llamó por su gracia, tuvo a bien revelar en mí a su Hijo' (Gál 1, 15-16)". ${ }^{12}$ O la propia María Zambrano, cuando dice: "Y así, al filo del oído, de los errores que nos hace cometer, podemos discernir esa última y secreta, indefinible esperanza que nos habita de ser llamados por nuestro nombre por alguien y aun por algo que no conocemos, de oírnos llamar de una vez por todas, una voz que nos procure la intima certeza de sabernos conocidos, conocidos del todo, enteramente identificados por alguien o por algo más allá de lo cotidiano."13

La vocación expresa por tanto la capacidad de entrega, del ser humano, es relación con un Tú, que es el Señor, que llama, y llama a través de un tú, de un maestro, de una compañía. Por eso los jóvenes lo que necesitan es un adulto que tenga un afecto por su vida, que se dirija al punto en que están no para convencerles (eso sería un engaño porque son dos libertades las que se encuentran y la adhesión tiene que ser de un yo presente para que la libertad se mueva ante la belleza de la realidad. El hombre busca la verdad, la desea en su corazón, por ello lo que se precisa es el despertar del yo que se expresa en la compañía. El educando necesita un maestro que le introduzca el mundo. En este sentido, la educación no es un proceso autónomo, sino que para educar hace falta una comunidad, una tradición, una concepción del bien común. No hay nada que inventar aqui, al contrario, lo que tengo que hacer es conocer lo mejor que me ofrece mi cultura, que no es ni una pieza de museo ni una ideologia, ni un ente neutral, pues requiere de un

12 Emmanuel Mounier, Personalismo y cristianismo, Obras Completas, I, Sígueme, Salamanca, 1992, pp. 873-874.

13 Maria Zambrano, Filosofia y educación, Ágora, Málaga, 2007, p. 58. 
yo vivo, que esté presente, con todas sus potencias, y que desee vivir a la altura de su tiempo. Por lo tanto, la relación educativa se construye sobre el encuentro vivo con la tradición, encuentro del que extraigo, del que aprendo, la forma de relacionarme con la realidad, con las cosas y con los demás, proporcionándome espacios significativos que el educando está llamado a descubrir. La tradición, que es una manera de introducir en la realidad ${ }^{14}$, que no busca homogeneizar, permite el ensanchamiento de las capacidades de relación, la propia creatividad expresada en el sujeto de una manera viva. Por lo tanto, requisito esencial para ser educador es haber puesto previamente en juego la propia libertad, para así conocer de primera mano, de forma viva, las joyas que ofrece la tradición. De esa manera ya se ha despertado en él el gusto por lo real, eso que el educando advierte como la pasión de un profesor, eso que atribuimos al maestro cuando decimos que tiene "autoridad", que significa hacer crecer, eso a lo que en otros ordenes educativos, humanos, llamamos testigo.

Una educación que no sea respuesta a la libertad concreta del educando, es decir de aquel a quien se quiere educar, es adiestramiento, pero no educación. Se adiestra a un perro para convertirlo en guardián de un rebaño, o en guardián de una casa, o incluso para trabajar en un aeropuerto o en una universidad, es decir, para que haga lo que nosotros queramos, para convertirlo en un instrumento de nuestro sistema social. Se adiestra a una persona porque no deseamos una educación que se dirija a su desarrollo concreto, según su propio destino, sino que deseamos que se convierta en un engranaje del Estado o del mercado. Así lo afirmó Edith Stein, pero también Emmanuel Mounier se sitúa en esta línea al defender que el fin de la educación no es "adiestrar al niño para una función o amoldarle a cierto conformismo, sino el de madurarle y armarle (a veces desarmarle) lo mejor posible para el descubrimiento de esta vocación que constituye su mismo ser y el centro de reunión de sus responsabilidades de hombre [...] Solo la persona encuentra su vocación y hace su destino. Ninguna otra persona, ni hombre ni colectividad, puede usurpar esta carga." ${ }^{15}$

14 Sobre ello vid. Luigi Giussani, Educar es un riesgo, Encuentro, Madrid, 1986.

15 Emmanuel Mounier, “¿Qué es el personalismo?”, Obras completas, I, op. cit., p. 630. 
La libertad es el elemento fundamental de toda relación educativa. Sin libertad no puede haber formación. Pero la libertad no es una tabula rasa, no es una libertad abstracta, sino una libertad que se despierta ante posibilidades concretas de lo real. La primera labor del educador por tanto, sea madre, padre, amigo, maestro, sacerdote, es provocar el despertar de la libertad ante el atractivo de lo real. Por eso es muy importante seleccionar los aspectos de la realidad que pueden ayudar a que la libertad se ponga en movimiento.

Educar, por tanto, no consiste en transmitir solo conocimientos técnicos, no se trata de acumular un saber de tipo enciclopédico, pero tampoco de un adiestramiento de las fuerzas para hacer del hombre el correcto ciudadano adaptado al sistema, ni tampoco de una explicación de la realidad de corte positivista que se limite a los simples hechos, deslavazada, sin sentido y reductiva.

Cuando al hombre le interesa un ámbito de la realidad, los datos más o menos eruditos ya no son solo datos o hechos, sino que "lo que cae bajo los sentidos es la expresión de un significado, que quiere ser entendido"16. De esta manera, la formación consiste principalmente en que se genere una manera de relacionarse con el mundo, con las cosas, en la que el educando amplíe el sentido de su vida y de lo real.

La profesión, entonces, no es algo distinto de la vocación, sino una concreción, la manera concreta de aportar lo que uno es al bien de la comunidad, lo que también se realiza según los parámetros existentes, por ello educar consiste, como dice Benedicto XVI, en educar para vivir con plenitud y aportar lo mejor de sí mismo al bien de la comunidad.

¿Cuál es según esto la actitud que mejor corresponde al educador? Si atendemos a lo que hemos dicho hasta el momento, no puede haber fórmulas educativas perfectas. El educador es un compañero de camino, aquel que conduce al educando al

16 Edith Stein, Ser finito y ser eterno. Ensayo de una ascensión al sentido del ser, en OC, III p. 626. 
descubrimiento de su propia vocación, aquel que despierta en él el deseo de una relación con la realidad mediada por los bienes que le pone delante. Por ello debe ser consciente de que su actividad tiene unos límites. El educador ofrece, provoca, cuestiona, acompaña, intenta que comparezca toda la humanidad del educando, que se ponga en juego toda su persona. Por ello una verdadera antropología de la educación ha de contar con los límites de la propia actividad educativa. Me estoy refiriendo al concepto de la individualidad. Atender a la individualidad, al destino propio del sujeto particular transforma el significado de la labor educativa. Un verdadero realismo, es decir, mirar a las cosas como son, y no transformarlas en caricaturas o esquemas mentales, debe percibir el misterio inabarcable que es la persona concreta.. Sin embargo, es muy importante comprender que la atención a la individualidad no significa caer en un relativismo o en un subjetivismo que haga imposible la tarea educativa. Significa que el hombre es irrepetible, significa fundamentalmente pasión por el destino del otro, sabiendo que el sujeto de la educación tampoco conoce su destino, y que educar es acompañarlo, para que descubra aquello a lo que está llamado, es decir, su vocación original. La individualidad, en este sentido, no puede comprenderse sino ligada al ámbito de la fe y de la experiencia religiosa. ${ }^{17}$

En este sentido, como tarea a la que nos llamaba Benedicto XVI, en el contexto de la nueva evangelización, sólo existe un camino que permita acompañar al otro en el despertar de su vocación: un afecto verdadero. Solo una mirada amorosa, que se corresponde plenamente con lo que hemos denominado pasión por el destino del otro puede permitir al educador acompañarle. Se trata de una aventura que requiere la presencia del yo, que el yo despierte de la apatía, que encuentre a alguien capaz de atraer el corazón, desafiar la razón y poner en movimiento la libertad, porque la libertad se mueve a partir de la atracción de la realidad y porque el hombre busca encontrar un significado al que adherirse que permita una unidad del yo que acoja tanto la vocación como la profesión.

17 Sobre el concepto de individualidad, vid. Marco Paolinelli, "Natura, spirito, individualità in Edith Stein", en Michele D'Ambra, Edith Stein: lo spirito e la santità, OCD, Roma, 2007, pp. 51-112. 
Pero en nuestros días, no solo carecemos de suficientes educadores que tengan una pasión por lo humano tal que se arriesguen en esta aventura, sino que los propios alumnos acuden a la escuela como si se les hubiese cercenado previamente la capacidad de asombrarse ante el mundo, o dicho de otra manera, como si hubiesen perdido el gusto por la vida. Hasta la mirada del educador rebota en ellos como si chocase contra una pared ${ }^{18}$. Por eso es necesario insistir en la importancia del entorno, de las circunstancias, de comprender no solo el momento en que se asiste al aula sino toda la vida del joven como parte de esta labor educativa.

Ahora bien, el educador -padre, madre, amigo, maestro, compañero, etc.- no cuenta para la labor educativa únicamente con sus propias fuerzas que aparecen como minúsculas frente a la grandeza del misterio de la vida individual, sino que cuenta también con la fuerza de la gracia. La vocación del hombre es una respuesta, no es una mera elección: Alguien ha hablado antes.

La fe permite al educador esperar que la gracia actúe en lo que excede a sus propias fuerzas y así poder transmitirlo a los que educa, desde su propio testimonio personal: "Así, la fe viva es propia de la razón y del corazón, obra de la voluntad, y acción. Quien enseña a que se suscite, enseña todas las energías. Pero sólo se la puede suscitar si se ponen en juego todas las fuerzas, no sólo por medio de una seca enseñanza del entendimiento, y tampoco por una „plenitud sentimental“, que produce fanatismo, sino por una formación religiosa que desde la plenitud de la propia vida religiosa conduce a las profundidades de la divinidad y sabe expresar a Dios en su amabilidad, enciende el amor y lo garantiza con las obras (...). Con ello el alma se abre a toda la plenitud del mundo (...), y así a una cantidad inagotable de elementos formativos que pueden entrar en ella, edificarla y transformarla"19.

La posibilidad de acoger esta fe solo es posible por la experiencia del amor de Dios sobre nuestras vidas. Ningún hombre nos conoce

18 Edith Stein, La estructura de la persona humana, en OC, IV, p. 25.

19 Edith Stein, "Fundamentos de la educación de la mujer", en OC, IV, p. 206. 
perfectamente como somos, ni nosotros tampoco, no podemos prefijar la meta de la educación, ni encerrar al hombre en ningún esquema previo. En realidad todo el trabajo del educador es un trabajo preparatorio ${ }^{20}$ : abrir el sentido del mundo para el educando, porque una razón que se toma en serio la realidad descubre que ella es metáfora de otra cosa, es participación en Dios. Pero eso no lo consigue enseñando sólo con palabras. "Para educar hombres verdaderos tiene que ser él mismo un hombre verdadero." Por eso afirma Edith Stein que el educador conduce al discípulo por el camino apropiado cuando le lleva al punto en que él, el discípulo, quiere vivir en el seguimiento de Cristo, cuando comprende la mirada amorosa que Dios tiene sobre su vida. Así afirma Stein: "quien pone su vida en las manos de Dios, puede estar seguro, y sólo él puede estar seguro, de que llegará a ser totalmente él mismo, es decir, que llegará a ser lo que Dios ha previsto para él personalmente" ${ }^{21}$. Ese es, en definitiva, el cumplimiento definitivo de la vocación.

Educar, en definitiva, es atender al misterio de lo humano concreto, aunque pueda intuirse cada vez más la grandeza de ese misterio y pueda cultivarse, cuidando la relación con Dios como relación con el destino, como deseo de ser-con-Dios. En ello reside la esperanza de la verdadera educación, que ningún Estado ni partido político puede arrebatar y que consiste en comprender que hay en el corazón de todo hombre una vocación, una llamada hacia el destino, desde la cual vivimos y afrontamos toda la realidad, toda la vida. 